Thorax (1967), 22, 431.

\title{
Mixed glandular and squamous-cell carcinoma of the bronchus
}

\author{
DAVID J. B. ASHLEY AND H. DUNCAN DAVIES \\ From the Pathology Department, Morriston Hospital, Swansea
}

\begin{abstract}
A group of 26 examples of lung carcinoma showing mixed squamous and glandular differentiation is described. The clinical and biological features of the lesions are intermediate between those of squamous-cell carcinoma and adenocarcinoma of the bronchus. It is suggested that bronchial carcinoma is a single entity in which the type of cell, whether glandular or squamous, plays a much smaller part in determining the biological behaviour than the anatomical site of the tumour and the degree of differentiation of its component cells. It is suggested that mixed adenosquamous tumours arise from bronchial epithelial cells in the process of metaplastic change from the columnar to the squamous form.
\end{abstract}

The majority of malignant epithelial neoplasms arising as primary growths in the lung and bronchi may be classified as squamous-cell carcinoma, adenocarcinoma, or undifferentiated carcinoma, as they show only one mode of differentiation. Occasionally a tumour is seen which shows histological features of both glandular and squamous type. The present paper is a study of the clinical and pathological behaviour of 26 such lesions in men which were seen in a histological survey of 707 cases of primary pulmonary malignancy. Reference was made in a previous communication (Ashley and Davies, 1967) to 39 such cases: on further detailed study of the sections from these lesions 13 were rejected as it was considered that they failed to attain the strictest diagnostic criteria.

\section{DIAGNOSTIC CRITERIA}

In all cases the tumours were infiltrating epithelial growths judged to have arisen in the epithelium of bronchial passages. All showed invasion of the supporting tissue of the lung and all showed hypertrophy and pleomorphism of tumour cells. In 10 there was invasion of mediastinal lymph nodes.

The diagnosis of the squamous element is based on the occurrence of a pattern of graded differentiation in blocks of tumour cells. In a zone adjacent to the connective tissue stroma of the lesion the cells are arranged with their axes at right angles to the stromatumour junction. They are at this level columnar in form (Figs 1, 2, and 3). As the distance from the stroma increases the cell axes tend to rotate so that they lie parallel to the stroma-tumour junction and the width of the cells relative to their length decreases (Fig. 1). Keratinization with the production of squamous and epithelial pearls is not regarded as an essential feature, although it did occur on occasion (Fig. 4).

The diagnosis of the glandular element is based on the presence of acinar spaces lined by cubical or columnar epithelium (Figs 2, 3, 4, 5, and 6). An important diagnostic feature is that the acinar space should be empty or should contain only mucoid debris (Figs 3, 4, and 6) or desquamated cuboidal cells (Figs 5 and 6 ). In poorly differentiated squamous carcinoma occasional rounded spaces surrounded by cuboidal cells are seen, but on close examination it is seen that these contain stromal cells and often blood vessels, so that the apparent central cavity is analogous to the connective tissue stalk of a papilloma rather than a gland space.

Glandular and squamous elements were usually intimately mixed in the tumours (Figs 2, 3, 4, and 6) ; occasionally, separate areas showed either squamous (Fig. 1) or glandular (Fig. 5) differentiation. In 13 instances the tumour was predominantly of squamous type with only small areas of glandular differentiation; in 10 instances it was predominantly of glandular type, and in three instances the two modes of differentiation were present in approximately equal amounts in the material available for examination.

Special staining techniques were not used as diagnostic criteria because, as will be mentioned below, P.A.S.-positive and Alcian blue-positive material was frequently found in lung cancers showing exclusively squamous differentiation. 


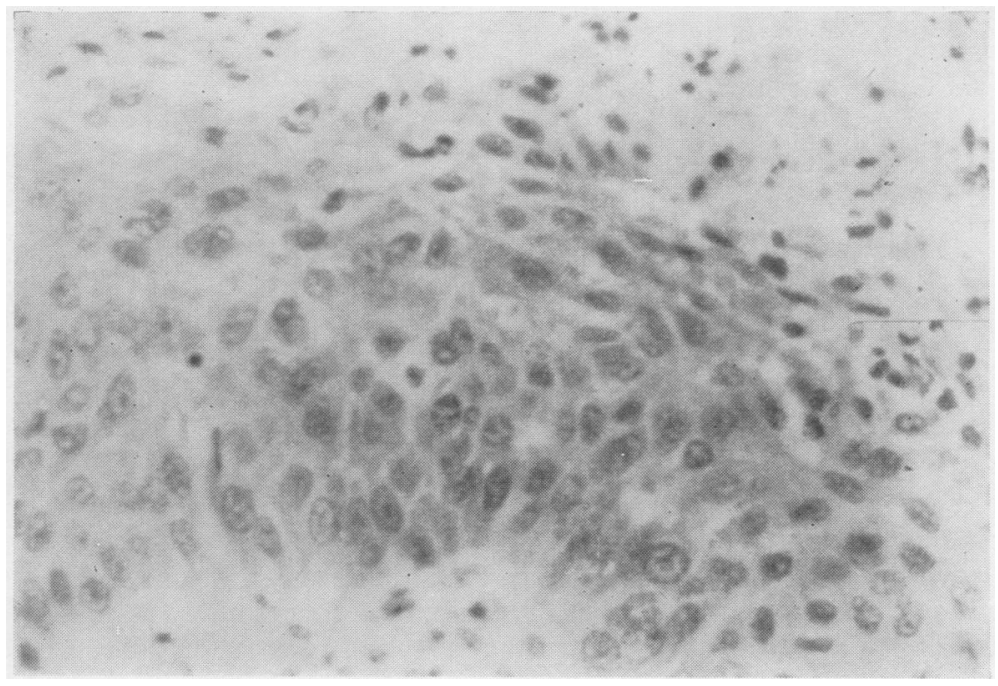

FIG. 1. The gradient of squamous differentiation from the stromal to the free side of a group of tumour cells $(360 / 57)$. H. and E. $\times 320$.

FIG. 2. Squamous differentiation with areas of glandular carcinoma $(415 / 60)$. H. and E. $\times 170$.

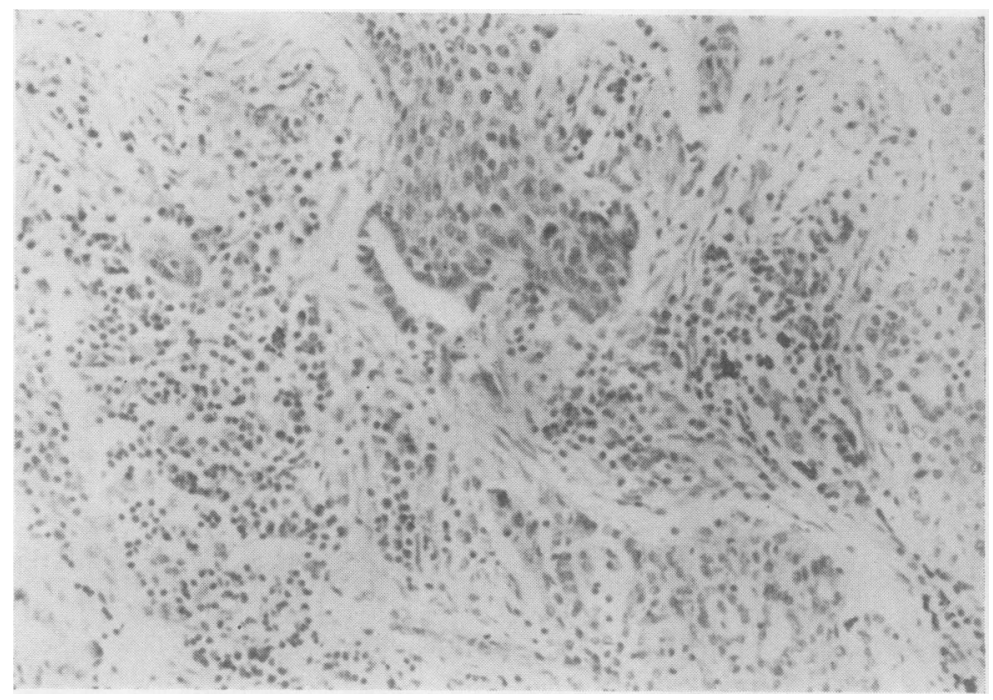

\section{CLINICAL FEATURES}

AGE Two men were less than 40 years of age, three between 40 and 50, 11 between 50 and 60 , and 10 between 60 and 70 years. The mean age at diagnosis was $56 \cdot 2$ years.

SMOKING Smoking histories were available in 19 men. One was a non-smoker, three smoked less than 10 cigarettes per day, nine smoked between 10 and 20 per day, three smoked between 20 and 30 per day, and three were simply recorded as cigarette smokers.

SYMPToms Thirteen patients had the symptom of cough at the time of diagnosis, four had dyspnoea,
10 had chest pain, 12 had coughed up blood on one or more occasions, and three had general symptoms such as malaise. One patient had a recurrent laryngeal nerve palsy due to secondary deposits of tumour and one was symptom-free. In this last case the diagnosis was made on routine radiological examination.

BRONCHOSCOPY In 11 men the tumour was visible at bronchoscopy, in 15 it was not.

OPERABILITY The lesion was resectable in 18 instances and was adjudged inoperable in the remaining eight. Of the 15 men in whom the lesion was not visible at bronchoscopy it was resectable in 12 instances. 


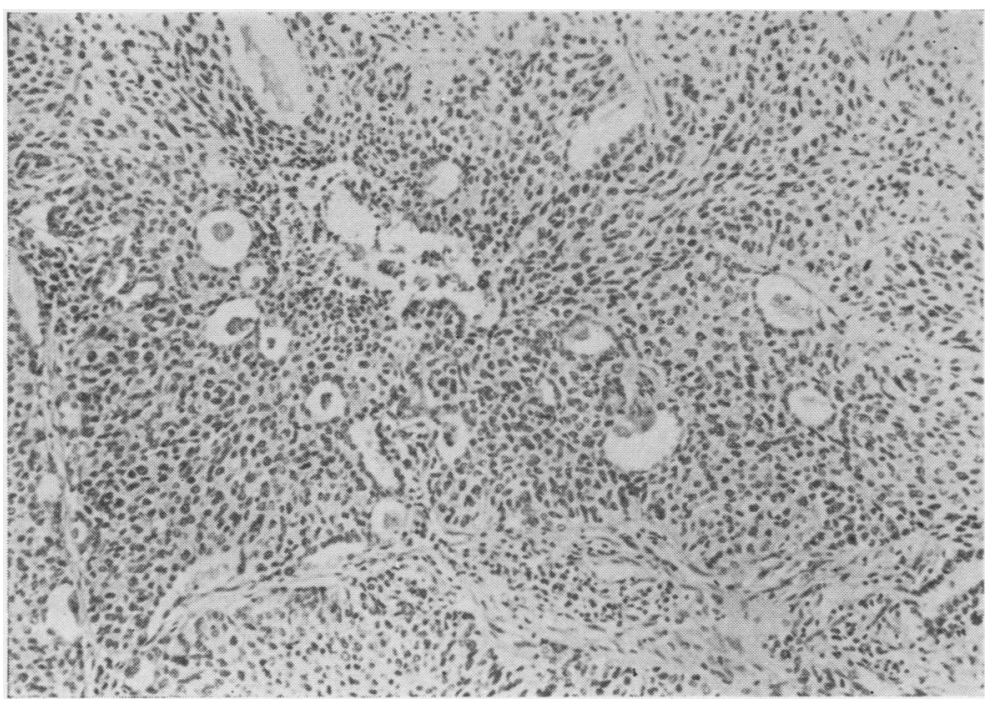

FIG. 3. A sheet of ill-differentiated squamous cells in which are many acini lined by cubical epithelium $(1,438 / 60)$. H. and $E . \times 110$.

FIG. 4. A neoplastic gland acinus adjacent to a focus of squamous carcinoma showing an epithelial pearl $(636 / 60)$. $H$. and $E$. $\times 320$.

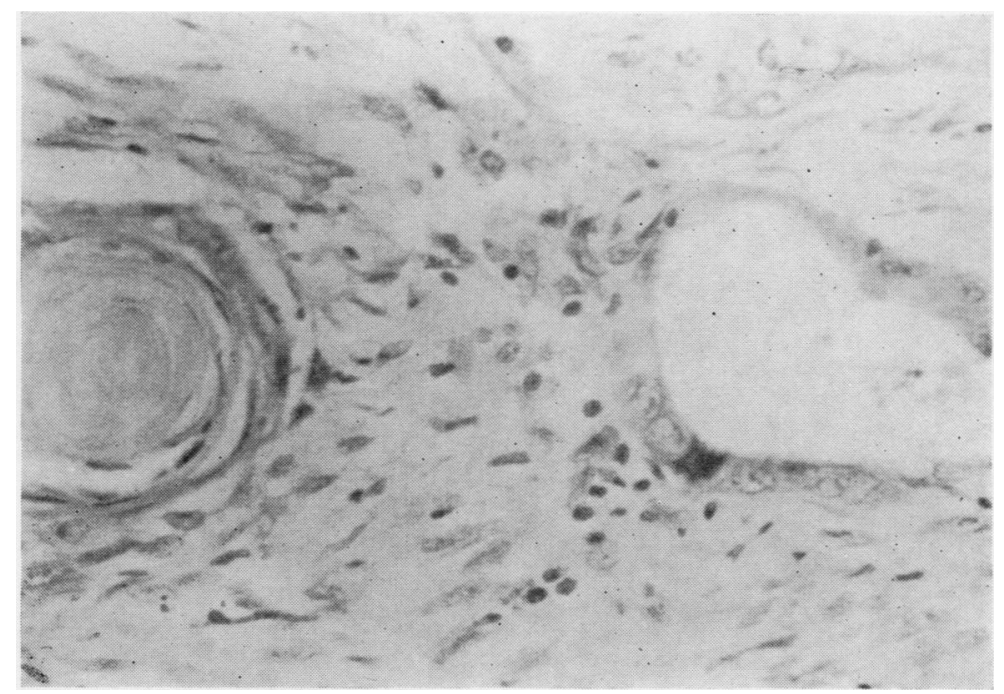

FOLLOW-UP Follow-up data were available for 13 patients who had survived thoracotomy for more than one month or whose chest had not been opened. Three of these patients were alive 12 months after the diagnosis had been made.

\section{DISCUSSION}

Mixed adenosquamous carcinoma of the lung has been described by many workers, usually with low frequency. Willis (1960) found six examples in his necropsy series of $84(7 \cdot 1 \%)$; Whitwell (1961) found $1.4 \%$ of such mixed tumours in bronchial biopsy material, $3 \%$ in material obtained at open operation, and $5.5 \%$ in necropsy material. Kreyberg (1962), however, regarded such mixed lesions as rare and found less than $1 \%$ to be of this type, while Walter and Pryce (1955) found $12 \%$ of lung cancers seen in necropsy material to be of this type and $19 \%$ of lung cancers in operation specimens. In our material, $26(3.7 \%)$ of 707 histologically proven lung cancers showed mixed glandular and squamous differentiation: in the total of 240 operable cases $7.5 \%$ were of this type. This proportion is of the same order as that found by most other workers and, as such large numbers of men suffer from lung cancer, mixed tumours of this 


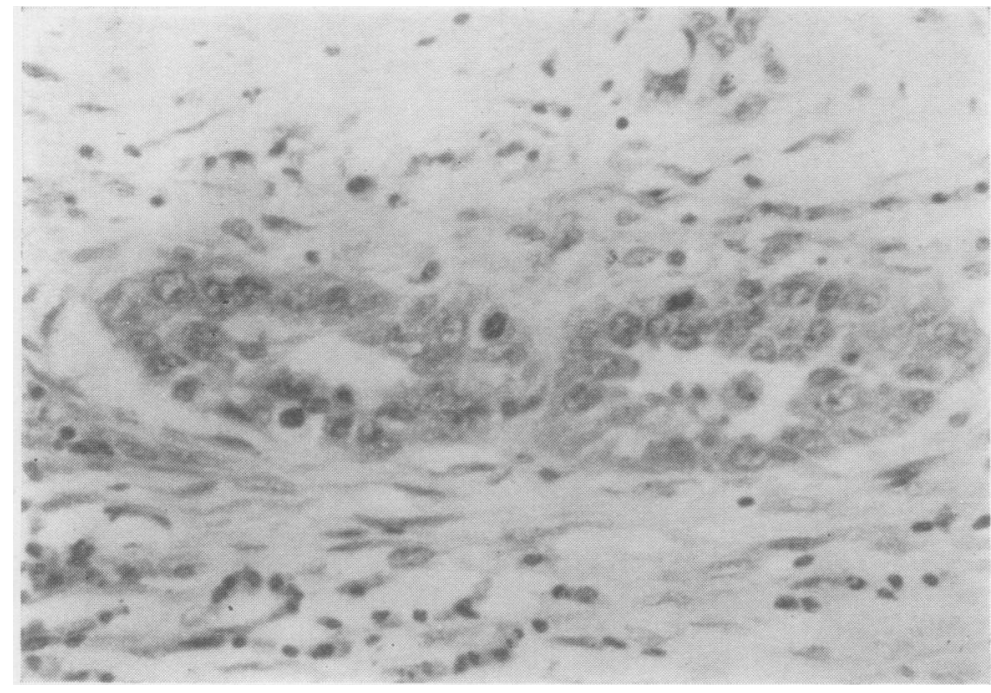

FIG. 5. The same tumour as in Figure 1, showing two neoplastic gland acini $(360 / 57) . H$. and $E$. $\times 320$.

FIG. 6. The detail of two acini lined by cubical epithelium in $a$ sheet of poorly differentiated squamous carcinoma $(1,438 / 60)$. $H$. and $E . \times 320$.

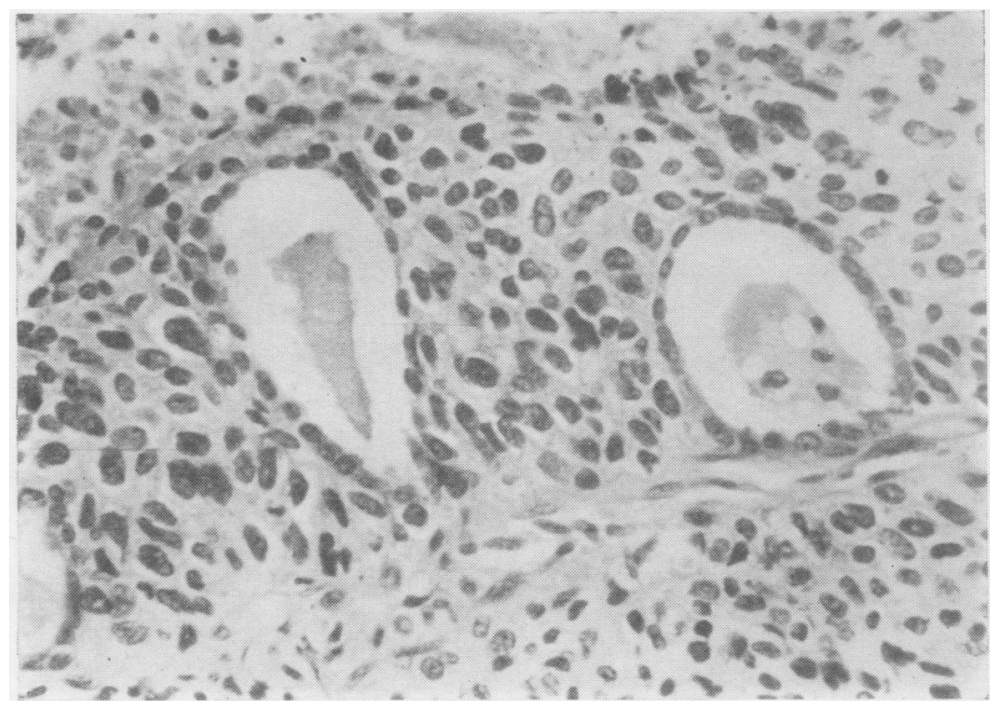

type form a considerable part of the load of neoplasia seen in man.

Consideration has been given at length to the biological behaviour of lung cancer of various histological types (Ashley and Davies, 1967). It is considered worth while here to relate the data in this group of cases to those obtained in the larger group, derived from the same population, of cases in whom only one mode of differentiation was seen. The age distribution of these 26 patients and the pattern of smoking habits did not differ significantly from those seen in the series of cancers with single lines of development.
The pattern of symptoms showed similarities to that of patients with both squamous carcinoma and adenocarcinoma. The frequency of diagnosis on routine radiological examination was lower than in well-differentiated squamous-cell carcinoma $(8.7 \%)$ or well-differentiated adenocarcinoma $(20.8 \%)$ and was comparable with that seen in less well-differentiated tumours. Cough was present less often than in patients with pure squamous carcinoma (68\%) and was commoner than in patients with pure adenocarcinoma $(52.5 \%)$. The frequency of dyspnoea was lower than in any of the five groups of lesions showing 
single line differentiation. This finding may, however, be related to the high frequency with which the diagnosis of mixed tumour was made on operation specimens, since patients with severe dyspnoea may be regarded as inoperable for this reason alone. Haemoptysis was commoner in association with squamous-cell tumours, whether well or poorly differentiated $(50.5 \% ; 46.2 \%)$, than in well or poorly differentiated adenocarcinoma or undifferentiated carcinoma $(25 \% ; 30.6 \%$; $33.4 \%)$. The frequency of this symptom in patients with these mixed tumours was $46 \%$, which corresponds to that found in the group with squamous differentiation. The frequency of chest pain, of symptoms of a general nature, and of symptoms due to secondary deposits showed no association with this histological type.

The frequency with which the lesion was visible at bronchoscopy $(42 \%)$ was intermediate between the frequencies found for squamous-cell carcinoma $(69 \cdot 5 \%)$ and adenocarcinoma $(28 \%)$.

Sixty-nine per cent of the lesions in the present series were regarded as operable. This proportion is higher than was seen in any of the groups of tumours with single lines of differentiation. It is, however, a false value, as the chance of detecting mixed modes of differentiation depends on the amount of tissue which is available to the histologist. The usual bronchial biopsy comprises a small fragment of the tumour and, while adequate for diagnosis, it is usually quite insufficient for the diagnosis of mixed tumour to be excluded. The proportion of bronchoscopically negative cases in whom the tumour proved to be operable $(80 \%)$ was the same as in patients with simple adenocarcinoma and a little higher than in patients with well and poorly differentiated squamous carcinoma (each $73 \%$ ).

It follows from the foregoing that mixed glandular and squamous-cell carcinoma of the lung occurs with a low frequency and that either the clinical behaviour does not differ significantly from that of lung neoplasms with a single mode of differentiation or, in its accessibility to bronchoscopy and the frequency of the major symptom (cough), it falls into an intermediate position between squamous-cell and glandular carcinomata. It was pointed out in our previous paper (Ashley and Davies, 1967) that lung cancer can be regarded as a single neoplastic entity in which the differences of clinical presentation and of prognosis can be related rather to the anatomical site of the lesion in the bronchial tree and to the degree of differentiation than to the mode of differentiation, whether epidermoid or glandular. The present series of cases shows that mixed squamous and glandular carcinoma of the lung also takes its place as one of the histological rather than biological variants of bronchial neoplasia.

The mode of origin of these tumours is not difficult to understand. The normal epithelial lining of the bronchial tree consists of a pseudo-stratified layer of ciliated columnar cells, some of which may retain the capacity to elaborate and to secrete mucus. When the bronchial epithelium is irritated it at first overacts, with the secretion of excessive mucus, but later undergoes metaplasia when the mode of differentiation of the cells is changed to that of a stratified squamous epithelium. This change, which is gradual rather than abrupt, is seen in the bronchial epithelium of cigarette smokers, who are accustomed to irritation of the mucosa (Auerbach, Stout, Hammond, and Garfinkel, 1961), and involves permanent or semipermanent changes in the method of expression of the full genotype of the individual which is locked in the gene sequences of the nuclear DNA of every cell. In normal differentiation some genes are suppressed and are never used in the lifetime of the cell ; others are free and are able to exercise their capacity to control the production of whatever proteins and polypeptides are relevant to the particular function which is being expressed by a particular cell at a particular time. Metaplasia, whether in epithelial or connective tissues, necessarily involves a change in the control of genetic expression by the cell, in this case a changeover from the genes which lead to a glandular form to the genes which lead to a squamous form. Usually the metaplastic changes are sufficiently well established to remain after neoplastic transformation has occurred, so that the familiar squamous-cell cancer is seen. Often carcinogenesis is accompanied by a loss of differentiation and the tumour cells show neither glandular activity nor squamous form: these lesions are the undifferentiated, small-cell carcinomata which exhibit a high degree of malignancy. We suggest that occasionally neoplastic transformation occurs in cells which are in the process of metaplasia and have not yet locked on to one or other mode of differentiation. Such tumours may show both glandular and squamous differentiation in their various parts. These tumours tend to be a little less accessible to the bronchoscope than the pure squamous tumours and a little less likely to be peripheral, and detectable on routine radiology. than the pure glandular tumours.

The morphology of neoplasms is not, of course, the only index of differentiation. Steiner and 
Friedell (1965) defined adenosquamous carcinoma of the cervix uteri as a tumour of squamous aspect in which mucin, detectable by P.A.S. staining or mucicarmine staining, was apparent. We took a group of 16 examples of squamous-cell carcinoma of the lung, none of which showed any evidence of glandular differentiation: 12 of these tumours showed P.A.S.-positive material and Alcian bluepositive material within tumour cells, suggesting that they had retained some of the capacities of the glandular cell of the bronchial epithelium despite their undoubted squamous form. Similar staining of a group of squamous-cell carcinomata of the skin showed no such material.

This work was carried out with the aid of a research grant from the Welsh Hospital Board.
We are indebted to our clinical colleagues, Dr. E. A. Danino, Dr. T. W. Davies, and Mr. C. J. Evans, for access to the clinical data.

\section{REFERENCES}

Ashley, D. J. B., and Davies, H. D. (1967). Lung cancer: histology $\vec{\circ}$ and biological behaviour. Cancer, 20, 165 .

Auerbach, O., Stout, A. P., Hammond, E. C., and Garfinkel, L. $\overrightarrow{\vec{H}}$ (1961). Changes in bronchial epithelium in relation to cigarette smoking and in relation to lung cancer. New Engl. J. Med., 265, 253. Kreyberg, L. (1962). Histological Lung Cancer Types. Norwegian
Universities Press, Oslo.

Steiner, G., and Friedell, G. H. (1965). Adenosquamous carcinoma or in situ of the cervix. Cancer, 18, 807.

Walter, J. B., and Pryce, D. M. (1955). The histology of lung cancer. $\vec{\omega}$ Thorax, 10, 107.

Whitwell, F. (1961). The histopathology of lung cancer in Liverpool. 음 Brit. J. Cancer, 15, 440.

Willis, R. A. (1960). Pathology of Tumours, 3rd ed. Butterworths, $\overrightarrow{-}$ London. 